\title{
A COMPUTER-ASSISTED DUAL WAVELENGTH MICRO- PHOTOMETRY SYSTEM FOR THE MEASUREMENT OF CYTOCHROME P-450 CONTENT IN SECTIONS
}

\author{
Jun Watanabe, Youko ASAKA, Tsuyoshi AMATSU and Shinsuke KANAMURA \\ Department of Anatomy, Kansai Medical University, Moriguchi, Osaka 570
}

Received for publication November 11, 1991

\begin{abstract}
To measure cytochrome P-450 (P-450) content in tissue sections, a dual wavelength microphotometry system linked with a personal computer was developed. A computer program to calculate $\mathbf{P}-450$ content in sections was also established. With this system, accuracy and microphotometric efficiency of the measurement of P-450 content in sections were improved markedly. Furthermore, this system can analyze many data derived from numerous microphotometric spots in sections using the program during measurement. Therefore, the measurement of $\mathbf{P}-\mathbf{4 5 0}$ content in tissue sections can be done precisely and rapidly with this system.
\end{abstract}

Cytochrome $\mathrm{P}-450$ (P-450) is an important drugmetabolizing enzyme. The reduced form of this enzyme binds carbon monoxide (CO), and produces a characteristic absorption band at $450 \mathrm{~nm}(1,3,6,7)$. Altman et al. (1) and Rappay et al. (6) observed microphotometrically the characteristic band of P450. Then, Gooding et al. (3) developed a microphotometric method for assay of P-450 content in sections. However, exact P-450 content can hardly be determined by the method of Gooding et al. $(2,6,7)$, because they used an inadequate extinction $\left(\Delta O D_{450}\right)$ instead of the true extinction $\left(\Delta \mathrm{OD}_{450-490}\right)$ and they could not minimize the effect of contaminating hemoproteins on the extinction of P-450. We therefore established a microphotometric method that accurately measures P-450 content in tissue sections (7), and developed an adequate microphotometry system (KWSP-1) (8). Then, we applied this method for the measurement of P-450 content in sections of livers from mice and rats $(5,7,8,10)$.

For precise determination of $\mathrm{P}-450$ content in tissue sections by our method, however, simultaneous measurement of percent transmission $(\% \mathrm{~T})$ values at

Correspondence to: Dr. Shinsuke Kanamura, Department of Anatomy, Kansai Medical University, 1 Fumizono-cho, Moriguchi, Osaka, 570 Japan.

This work is dedicated to Professor Kazuo Ogawa in memory of his retirement.
450 and $490 \mathrm{~nm}$ must be repeated many times from one microphotometric spot (8). In addition, many small spots must be measured within a portion in tissue section to avoid systematic distributional error resulting from heterogeneous distribution of $\mathrm{P}-450$ within the portion (8). Consequently, many measurements are required for determination of $\mathrm{P}-450$ in tissue sections. Furthermore, many data thus obtained must be processed according to the complicated calculation procedure (7). Therefore, a computerassisted microphotometry system and an effective computer program for processing these many data are necessary to improve the microphotometric accuracy and efficiency of measurement of P-450 content in sections.

In the present study, therefore, we developed a computer-assisted microphotometry system by linking a dual wavelength microphotometer with a personal computer. We also established a computer program for calculation of $\mathrm{P}-450$ content in tissue sections during the measurement.

\section{MATERIALS AND METHODS}

Twenty male ddY mice, 8 weeks old, were used. The animals were fed laboratory chow and water ad libitum. Under sodium pentobarbital anesthesia, livers of the animals were perfused with saline for $3 \mathrm{~min}$ via the portal vein $(5 \mathrm{ml} / \mathrm{min})$. Small blocks 
were cut from the left lobe of liver and frozen at $-80^{\circ} \mathrm{C}$. The remaining liver tissues were homogenized with $1 \mathrm{mM}$ Tris- $\mathrm{HCl}$ buffer containing $0.25 \mathrm{M}$ sucrose $\left(\mathrm{pH} \mathrm{7.4)}\right.$ at $4^{\circ} \mathrm{C}$. Then, cell lysates were prepared from the homogenates using Triton X-100 as described previously (9).

$\mathrm{P}-450$ content in the lysates was measured by the conventional biochemical difference spectrophotometry in a spectrophotometer (Hitachi, U-3,200, Tokyo, Japan) (7), or measured microphotometrically by the four-spectrum method using cuvette slides (7, 8). In short, the cell lysate was diluted with $0.1 \mathrm{M}$ Tris- $\mathrm{HCl}$ buffer $(\mathrm{pH} \mathrm{8.0)}$ and divided into four test tubes (tubes A, B, C and D). Then, CO was passed through the contents in tubes $\mathrm{B}$ and $\mathrm{D}$, and sodium dithionite $(5 \mathrm{mg} / \mathrm{ml})$ was added to the contents in tubes $\mathrm{C}$ and D. The contents in tubes $\mathrm{A}$ and $\mathrm{B}$ were separately poured into two cuvettes set on one glass slide, and the contents in tubes $\mathrm{C}$ and $\mathrm{D}$ were poured into two cuvettes set on another slide. The slides were set in a microphotometry system and twenty portions were selected at random from one cuvette. Then, percent transmission $(\% \mathrm{~T})$ values were recorded at $\mathbf{4 5 0}$ and $490 \mathrm{~nm}$ with a spot size of $1 \mu \mathrm{m}$ at $5 \mathrm{~min}$ after the setting.

For the measurement of P-450 content in tissue sections, four serial frozen sections, $4,6,8,10,13$ or $16 \mu \mathrm{m}$ in thickness, were cut from one block, picked up on coverslips, and separately incubated in $10 \mathrm{ml}$ of an incubation medium $(50 \mathrm{mM}$ Tris- $\mathrm{HCl}$ buffer containing $10 \%$ sucrose, $\mathrm{pH} 8.0$ ) (medium $\mathrm{A}$ ) alone, in the medium saturated with CO (medium B), in the medium containing sodium dithionite $(5 \mathrm{mg} / \mathrm{ml})$ (medium $\mathrm{C}$ ), and in the medium saturated with $\mathrm{CO}$ and containing sodium dithionite (medium D) for $1 \mathrm{~min}$ at room temperature (7). The sections incubated in the medium A or B were taken onto one glass slide and the sections incubated in the medium $\mathrm{C}$ or $\mathrm{D}$ were taken onto another slide. The sections were separately mounted in the same incubation medium, and set in the microphotometry system. Then, \% $\mathrm{T}$ values were recorded at 450 and $490 \mathrm{~nm}$ with a spot size of $1 \mu \mathrm{m}$ at $5 \mathrm{~min}$ after the setting.

Results were subjected to statistical analysis using the F-test and unpaired Student's t-test. Spearman's rank order correlation coefficient was used for ascertaining the relationship between actual measurement and expected value calculated from the content measured by biochemical spectrophotometry.

\section{RESULTS}

\section{Computer-assisted microphotometry system}

Hardware configuration. The developed system, KWSP-2, is comprised of a dual monochrometer-and dual photomultiplier-equipped microphotometer (KWSP-1) (8), an autostage (Auto Scanning Stage, Nikon, Tokyo, Japan), and a personal computer (PC 9801-DX2, NEC, Tokyo, Japan). To stabilize intensity of incident light beam, a direct current voltage stabilizer (PR-657, Trio, Tokyo, Japan) is connected with an alternating current voltage stabilizer (SLIDAC S110, Tokyo Shibaura Electric, Tokyo,

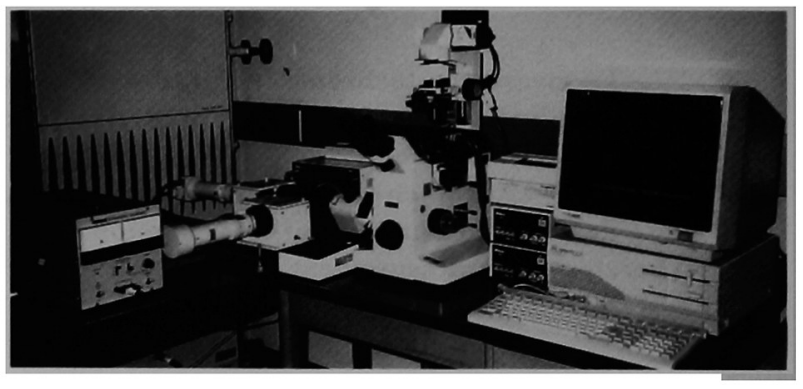

Fig. 1. Appearance of KWSP-2.

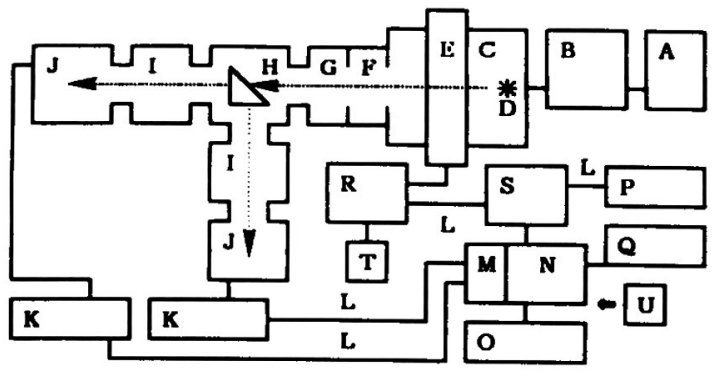

FIG. 2. Schematic diagram of KWSP-2. A, alternative current voltage stabilizer (SLIDAC S110); B, direct current voltage stabilizer (PR-657); C, inverted microscope with Nomarski differential interference contrast system (TMDNTF, Nikon, Tokyo); D, lump; E, autostage (Auto scanning stage); F, aperture (TDC-T, Nikon); G, shutter (PFX, Nikon); H, prism (SS1, Kogaku, Osaka); I, monochromators (CT-10S, Japan Spectroscopic, Tokyo); J, photomultipliers (R-374, Hamamatsu Photonics, Hamamatsu); K, photometers (P1, Nikon); L, RS-232G cables; M, interface board (PC9861K, NEC, Tokyo); N, computer (PC9801-DX2); O, keyboard; P, printer (P-40, EPSON, Tokyo); Q, display (PC8853N, NEC); R, autostage control unit (Nikon); S, data transfer switch (DTS2-232, ELECOM, Osaka); T, joystick; U, softwear (P45MS-SQ). Parts B-D and F-K are components of KWSP-1. Parts A, E, and L-U are newly-equipped components. Pathways of light beam are shown as broken lines. The beam passes in the direction of arrowheads. 
Japan) in this system. The microphotometer is interfaced to the computer through RS-232C cables. The appearance and schematic diagram of the system are shown in Figs. 1 and 2, respectively. This system can measure simultaneously $\% \mathrm{~T}$ values of specimens at 450 and $490 \mathrm{~nm}$ at a bandwidth of $0.5 \mathrm{~nm}$. With this system, 100 simultaneous measurements can be done within $1 \mathrm{sec}$, and the $\% \mathrm{~T}$ values thus measured are averaged automatically. Then, the averaged values are transferred to the computer through the RS-232C cables under a communication command of newly established computer program as described below.

Software. A computer program established for the measurement and calculation of P-450 content in the present study (P45MS-SQ) is written in MS-DOS N88BASIC (86). This program is comprised of an opening menu plus equipment warming up section and a main (measurement and calculation) section. In the program, three communication files are prepared for data transportation via RS-232C cables. Communication speed is set at 1,200 bits per sec.

Measurement and calculation of $P-450$ content with KWSP-2

Before the measurement, microphotometric parameters (magnification, spot size, iniensity of incident light beam and dynode voltages of photomultipliers) were set according to indications shown in the cathode ray tube of the computer. The equipment became ready to start the measurement at $10 \mathrm{~min}$ after the setting. Then, the measurement and computations were carried out as follows.

1) Two sections incubated separately in the medium $\mathrm{C}$ or $\mathrm{D}$ were set on the stage of the microphotometer. The $\% \mathrm{~T}$ from glass slides were measured and transferred to the computer. The values were averaged as a blank for $100 \%$ transmission $(100 \% \mathrm{~T})$ using the program.

2) Apparent $\% \mathrm{~T}$ values at 450 and $490 \mathrm{~nm}$ were measured simultaneously from a spot in a selected portion of section $\mathrm{D}$ and transferred to the computer. In the computer, the apparent values were converted into absorbances as follows:

$$
\begin{aligned}
& \text { absorbance (OD) }= \\
& \quad-\log (\text { apparent } \% \mathrm{~T} / 100 \% \mathrm{~T})
\end{aligned}
$$

Then, the absorbances at $450 \mathrm{~nm}$ or those at $490 \mathrm{~nm}$ measured in the spot were averaged, and the difference of absorbance at $450 \mathrm{~nm}$ minus that at $490 \mathrm{~nm}$ in the spot was calculated.

3) Apparent $\% \mathrm{~T}$ values at 450 and $490 \mathrm{~nm}$ were measured in other spots within the portion of section $D$. The data were separately processed in the computer as described above, and the resulting values were averaged. The averaged value was defined as $\mathrm{D}_{450-490}$.

4) Apparent $\% \mathrm{~T}$ values at 450 and $490 \mathrm{~nm}$ were measured in spots within corresponding portion of section $\mathrm{C}$, processed as above, and the value $\mathrm{C}_{450-490}$ was calculated.

5) Two sections incubated separately in the medium A or B were set on the stage of the microphotometer, and the $100 \% \mathrm{~T}$ value was measured as described in 1).

6) Apparent $\% \mathrm{~T}$ values at 450 and $490 \mathrm{~nm}$ were measured in spots in corresponding portions of sections $\mathrm{A}$ and $\mathrm{B}$, processed separately as above, and the values $A_{450-490}$ and $B_{450-490}$ were calculated.

7) The extinction of P-450 $\left(\Delta O D_{450-490}\right)$ was calculated from the results from four serial sections $A, B, C$, and $\mathrm{D}$ using the following formula:

$$
\begin{gathered}
\Delta \mathrm{OD}_{450-490}=\left(\mathrm{A}_{450-490}+\mathrm{D}_{450-490}\right) \\
-\left(\mathrm{B}_{450-490}+\mathrm{C}_{450-490}\right) \cdots \cdots \cdots \cdots
\end{gathered}
$$

8) The resulting extinction of $\mathrm{P}-450$ was converted into the content using molar extinction coefficient as follows:

$$
\begin{aligned}
& \text { Concentration }(\mathrm{M})= \\
& \left(\Delta \mathrm{OD}_{450-490}\right) / \mathrm{EC} \cdot(\mathrm{D} / \mathrm{d})
\end{aligned}
$$

where $\mathrm{EC}=$ extinction coefficient of P-450 $(91,000$

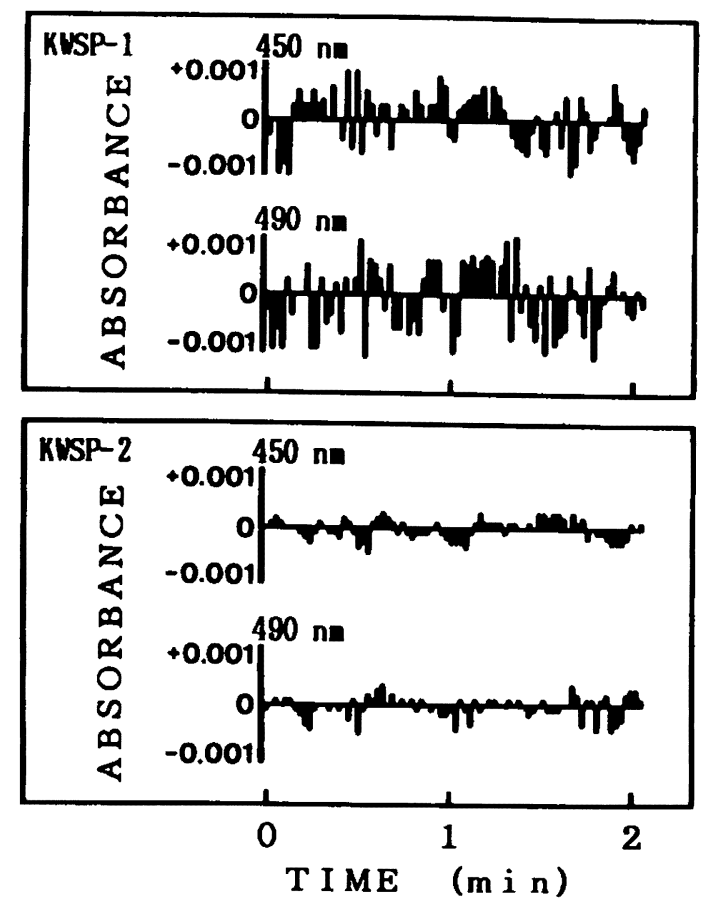

Fig. 3. Stability of intensity of incident light beam. Absorbances were measured in a neutral density filter with a spot size of $1 \mu \mathrm{m}$ using KWSP-1 (A) or KWSP-2 (B). 


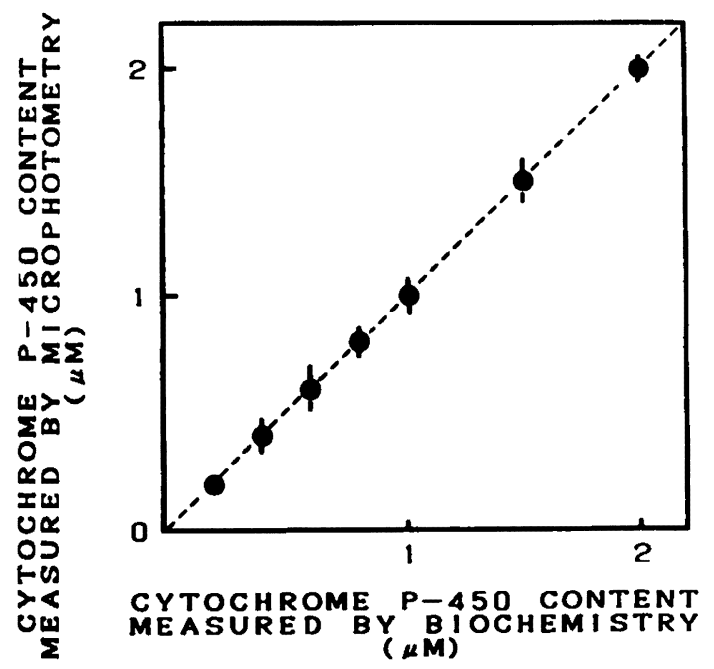

Frc. 4. Relationship between cytochrome P-450 content in diluted cell lysate from livers of adult mice as measured by microphotometry (actual measurements) and those estimated by biochemical difference spectrophotometry (expected values). Microphotometric values were measured in diluted lysates in cuvette slides by the four-spectrum method using KWSP-2 with a spot size of $1 \mu \mathrm{m}$. Values are means $\pm \mathrm{S}$. D. for five repeated experiments.

liter $\left.\mathrm{mol}^{-1} \cdot \mathrm{cm}^{-1}\right), \quad \mathrm{D}=1 \mathrm{~cm}, \quad$ and $\mathrm{d}=$ section thickness.

Stability of the intensity of incident light beam

The noise and fluctuations in incident light beam

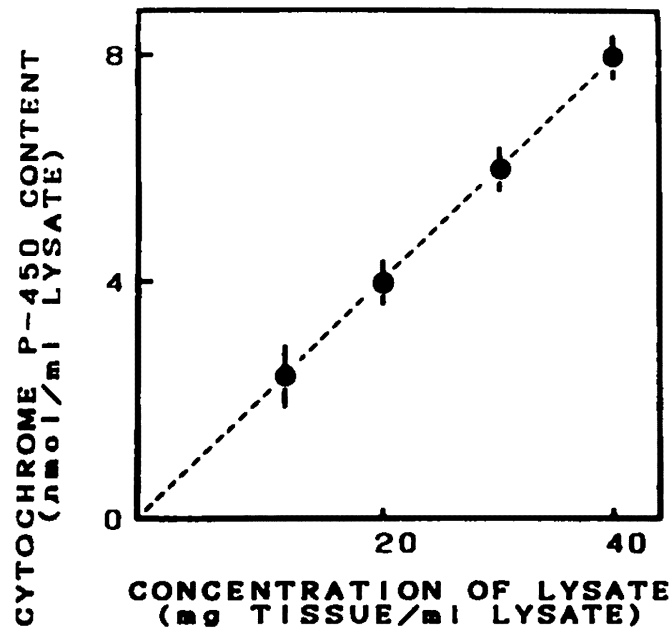

Fig. 5. Relationship between content of cytochrome P-450 in diluted cell lysates from adult mice and concentration of the diluted lysates. Content of cytochrome P-450 in the diluted lysate were measured by four-spectrum method with KWSP2 with a spot size of $1 \mu \mathrm{m}$. Values are means $\pm \mathrm{S}$. D. for five repeated experiments.

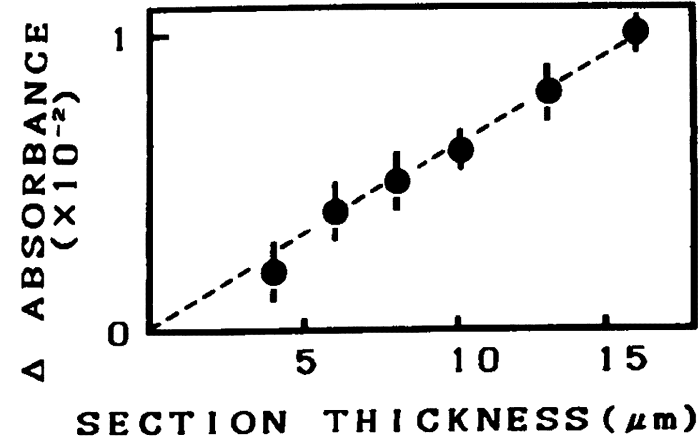

Fig. 6. Relationship between the extinction of cytochrome P450 (difference of absorbance at $450 \mathrm{~nm}$ minus that at $490 \mathrm{~nm} ; \Delta$ absorbance) and section thickness. Corresponding fields containing midzonal hepatocytes in serial sections from the liver of an adult mouse were analyzed. Values are means $\pm S$. D. for four repeated experiments.

diminished in KWSP-2; the value of KWSP-2 was about one-fourth the value of KWSP-1 (Figs. 3A, 3B). Accuracy of the measurement

When the amount of P-450 in diluted cell lysate measured with KWSP-2 by the four-spectrum method was plotted as a function of the expected value calculated from the amount in lysate measured by biochemical analysis, there was a close relation (correlation coefficient $r>0.97$ ) between the two values (Fig. 4). If one spot was measured 5 times (500 measurements $/ 5 \mathrm{sec}$ ), KWSP-2 can measure $\triangle \mathrm{OD}_{450-490}$ of 0.001 . Therefore, the limit of sensitivity is calculated to be less than $90 \mathrm{nM}$ of P-450 at $1 \mathrm{~mm}$ of lightpass and a spot size of $1 \mu \mathrm{m}$ by the use of KWSP2 , while the value of KWSP-1 is $300 \mathrm{nM}(8)$.

When the content of P-450 in diluted cell lysate measured with KWSP-2 by the four-spectrum method was plotted as a function of concentration of the lysate, there was a linear relation between the content of P450 and concentration of the lysate (Fig. 5). When the content of P-450 in liver section measured with KWSP-2 was plotted as a function of section thickness, there was a linear relation between the content and section thickness (Fig. 6). Therefore, Beer-Lambert's law holds for our microphotometric method measured with KWSP-2.

\section{DISCUSSION}

As shown in the present study, we developed KWSP-2, a computer-assisted microphotometry system, and a computer program for calculating P-450 content in tissue sections. P-450 content in liver sections can be accurately measured with this system. 
Furthermore, the system minimizes the time taken to calculate the P-450 content.

For precise determination of P-450 content in tissue sections, measurement must be repeated many times for each microphotometric spot (8). Furthermore, to reduce systematic distributional error, many small spots must be measured in tissue sections $(4,8)$. On the other hand, our previous study revealed that the extinction of $\mathrm{P}-450$ bound with $\mathrm{CO}$ is stable within $25 \mathrm{~min}(7)$, indicating that the measurement must be done within $25 \mathrm{~min}$. Therefore, the microphotometric efficiency is important for obtaining accurate results. KWSP-2 can measure at least 1,200 spots within $25 \mathrm{~min}$ with a spot size of $1 \mu \mathrm{m}$, although KWSP-1 can measure only 400 spots within 25 min with a spot size of $2 \mu \mathrm{m}$ (7).

There are various random errors which must be considered in microphotometric measurements (4). The errors result from fluctuations in light output and from variations in optical noise. These random errors can be reduced by stabilizing intensity of incident light beam impinging onto sections and/or by repeating measurements for each microphotometric spot. The intensity of light beam tends to change according to fluctuations in voltage in lump circuit. The noise and fluctuations in light output can thus be reduced by linking an alternating current voltage stabilizer with a direct current voltage stabilizer in KWSP-2. Consequently, optical noise and fluctuations in light output during the measurement diminished in KWSP-2. Furthermore, the random errors can also be reduced by repeating measurements, because the microphotometric efficiency of KWSP-2 is high. In addition, the lower limit of sensitivity of the four-spectrum method measured with KWSP-2 $(90 \mathrm{nM} / \mathrm{mm}$ lightpass) was about 3 times greater than the value of the method measured with KWSP-1 $(300 \mathrm{nM} / \mathrm{mm}$ lightpass). Therefore, microphotometric accuracy for the measurement of P-450 was improved markedly by the use of KWSP-2.

The process of calculation for obtaining P-450 contents is time consuming. For example, if 40 portions in a series of serial sections (4 measurements/spot, 160 spots/4 serial sections) were analyzed, a total of 1,280 apparent $\% \mathrm{~T}$ values at 450 and $490 \mathrm{~nm}$ were measured, and it took $40-50 \mathrm{~min}$ to process these data with a calculator. On the other hand, the P45MS-SQ program eatablished in the present study runs under time-sharing operation system which can process most of the data during the measurement. Therefore, it takes only two minutes for calculating and printing the data plus storing the data into a floppy disk.
The P45MS-SQ program is written in MS-DOS BASIC. The data measured with KWSP-2 can thus be directly introduced into commercially available MS-DOS softwares for statistical analysis. Furthermore, the autostage can be controlled by MS-DOS BASIC program. The fully automated measurement of $\mathrm{P}-450$ content in tissue sections is therefore possible using KWSP-2. In addition, KWSP-2 can be applied to the measurement of hemoglobin content in sections according to the method of Hutton (4) with slight modification of the computer program.

\section{REFERENCES}

1. Altman, F. P., Moore, D. S. and Chayen, J.: The direct measurement of cytochrome $\mathrm{P} 450$ in unfixed tissue sections. Histochemistry 41; 227-232, 1975.

2. Chayen, J., Bitensky, L., Johnstone, J.J., Gooding, P. E. and Slater, T.F.: The application of microspectrophotometry to the measurement of cytochrome P450. In "Quantitative cytochemistry and its applications", ed. by J. R. Pattison, L. Bitensky and J. Chayen, Academic Press, London, 1978, pp.129-137.

3. Gooding, P. E., Chayen, J., Sawyer, B. and Slater, T. F.: Cytochrome P-450 distribution in rat liver and the effect of sodium phenobarbitone administration. Chem. Biol. Interactions 20; 299-310, 1978.

4. Hutton, R. D.: The measurement of intracellular hemoglobin. In "Quantitative cytochemistry and its applications", ed. by J. R. Pattison, L. Bitensky and J. Chayen, Academic Press, London, 1978, pp.139-147.

5. Kanai, K., Watanabe, J. and Kanamura, S.: Microphotometric analysis of cytochrome P-450 in periportal, midzonal, and perivenular hepatocytes of mice treated with phenobarbital. J. Histochem. Cytochem. 38; 1599-1605, 1990.

6. Rappay, G. Y., Bacsy, E. and Stark, E.: Microspectrophotometry of mitochondrial cytochrome $\mathrm{P}-450$ in single adrenal cells. Histochem. J. 8; 283-290, 1976.

7. Watanabe, J., Kanai, K. and Kanamura, S.: A new microphotometric method for measurement of cytochrome $\mathrm{P}-450$ in sections of liver. $J$. Histochem. Cytochem. 37; 1257-1263, 1989.

8. Watanabe, J. and Kanamura, S.: An improved microphotometry system for measurement of cytochrome P-450 in hepatocyte cytoplasm. J. Histochem. Cytochem. 39; 689-694, 1991.

9. Watanabe, J., Kanai, K. and Kanamura, S.: Measurement of NADPH-ferrihemoprotein reductase content in sections of liver. J. Histochem. Cytochem. 39; 1635-1643, 1991.

10. Watanabe, J., Asaka, Y., Kanai, K. and Kanamura, S.: Relation between cytochrome P-450 increase and endoplasmic reticulum proliferation in hepatocytes of mice treated with phenobarbital. A microphotometric and morphometric study. J. Histochem. Cytochem. in press. 